\title{
Presence of periaortic gas in Clostridium septicum-infected aortic aneurysm aids in early diagnosis: a case report and systematic review of the literature
}

Fumihito Ito ${ }^{1 *}$, Ryota Inokuchi' ${ }^{2,3}$, Akinori Matsumoto ${ }^{1}$, Yoshibumi Kumada ${ }^{1}$, Hideyuki Yokoyama' ${ }^{1}$, Tokiya Ishida', Katsuhiko Hashimoto ${ }^{1}$, Masashi Narita ${ }^{4}$ and Kazuaki Shinohara ${ }^{1}$

\begin{abstract}
Background: Clostridium septicum-infected aortic aneurysm is a fatal and rare disease. We present a fatal case of $C$. septicum-infected aortic aneurysm and a pertinent literature review with treatment suggestions for reducing mortality rates.

Case presentation: A 58-year-old Japanese man with an unremarkable medical history presented with a 3-day history of mild weakness in both legs, and experienced paraplegia and paresthesia a day before admission. Upon recognition of signs of an abdominal aortic aneurysm and paraplegia, we suspected an occluded Adamkiewicz artery and performed a contrast-enhanced computed tomography scan, which revealed an aortic aneurysm with periaortic gas extending from his chest to his abdomen and both kidneys. Antibiotics were initiated followed by emergency surgery for source control of the infection. However, owing to his poor condition and septic shock, aortic repair was not possible. We performed bilateral nephrectomy as a possible source control, after which we initiated mechanical ventilation, continuous hemodialysis, and hemoperfusion. A culture of the samples taken from the infected region and four consecutive blood cultures yielded C. septicum. His condition gradually improved postoperatively; however, on postoperative day 10, massive hemorrhage due to aortic rupture resulted in his death.

Conclusions: In this patient, C. septicum was thought to have entered his blood through a gastrointestinal tumor, infected the aorta, and spread to his kidneys. However, we were uncertain whether there was an associated malignancy.

A literature review of $C$. septicum-related aneurysms revealed the following: 6-month mortality, 79.5\%; periaortic gas present in $92.6 \%$ of cases; no standard operative procedure and no guidelines for antimicrobial administration established; and C. septicum was associated with cancer in $82.5 \%$ of cases.

Thus, we advocate for early diagnosis via the identification of periaortic gas, as an aortic aneurysm progresses rapidly. To reduce the risk of reinfection as well as infection of other sites, there is the need for concurrent surgical management of the aneurysm and any associated malignancy. We recommend debridement of the infectious focus and in situ vascular graft with omental coverage. Postoperatively, orally administered antibiotics must be continued indefinitely (chronic suppression therapy).

We believe that these treatments will decrease mortality due to C. septicum-infected aortic aneurysms.
\end{abstract}

Keywords: Infected aortic aneurysm, Aortic rupture, Aortic dissection, Sepsis, Septic shock, Clostridium spp, Colon adenocarcinoma, Adult

\footnotetext{
* Correspondence: fleurs.de.cerisier87@gmail.com

${ }^{1}$ Department of Emergency and Critical Care Medicine, Ohta Nishinouchi

Hospital, 2-5-20 Nishinouchi, Koriyama, Fukushima 963-8558, Japan

Full list of author information is available at the end of the article
} 


\section{Background}

Clostridium septicum is an anaerobic, spore-forming, toxin-producing Gram-positive bacillus with peritrichous flagella that only accounts for $1.3 \%$ of all clostridial infections. However, the sepsis caused by this organism is severe and has an overall mortality rate of approximately $60 \%$ [1]. The clinical relevance of this form of sepsis lies in its association with gastrointestinal malignancy, with the most common being adenocarcinoma of the right colon [2-4]. It is thought that this organism enters the blood through gastrointestinal tumors and infects the aorta.

Although C. septicum is a known cause of gas gangrene, it is a very rare cause of an aortic aneurysm. Why this organism favors aortic infection and how clostridial mycotic aneurysms form are not fully understood. It is thought that ulcerative lesions of the gastrointestinal tract, especially colon carcinoma, allow the organism to enter the bloodstream, followed by seeding of an atheromatous focus in the aorta [5]. Seeding of the aorta by this organism can often lead to the rapid development of an infected aneurysm, which is uniformly fatal in the absence of surgical intervention [6, 7]. Here we present a fatal case of C. septicum-infected aortic aneurysm. To enable the early diagnosis and identification of effective treatment options, we review cases of $C$. septicum-infected aortic aneurysm from the literature.

\section{Case presentation}

A 58-year-old Japanese man complained of mild weakness in both legs 3 days prior to hospitalization. He was admitted to our hospital because he was unable to move his legs and had lost sensation in both legs 1 day prior to hospitalization. He had no remarkable medical history. Although he was lucid, he had a blood pressure of 99/67 mmHg, pulse of 110 beats per minute (bpm), body temperature of $36.1{ }^{\circ} \mathrm{C}$, respiratory rate of 28 breaths/minute, and paralysis and numbness below the T11 level. Because we recognized signs of an abdominal aortic aneurysm and paraplegia on physical examination, we suspected an occlusion of the artery of Adamkiewicz and so performed a contrast-enhanced computed tomography (CT) scan. It revealed the formation of an aneurysm with gas in the aortic wall extending from his chest to his abdomen as well as in both kidneys (Fig. 1); no malignant tumor was detected. Lumbar magnetic resonance imaging also revealed a spinal cord infarction below the T11 level. We initiated antibiotic therapy with $9 \mathrm{~g} /$ day ampicillin/sulbactam, $1800 \mathrm{mg} /$ day clindamycin, and $120 \mathrm{mg} /$ day gentamycin; during this period, metronidazole was not available for intravenous injection in Japan. We then performed an emergency surgery for source control of the infection. However, owing to his

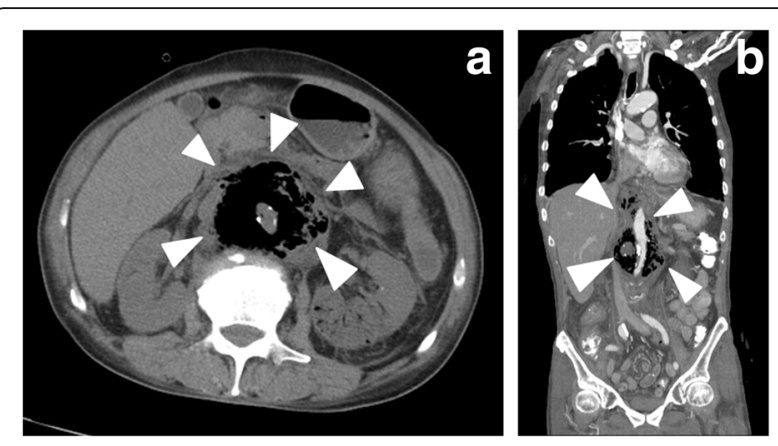

Fig. 1 a Axial view, and b coronal view contrast-enhanced computed tomography scan images showing an aneurysm with periaortic gas (the area immediately outside the gas-filled region is the tunica adventitia of the aorta; shown by arrowheads)

poor general condition and septic shock, surgery for the mycotic aneurysm was deemed impossible. Thus, we performed bilateral nephrectomy as a source control, and postponed the surgery for the aortic aneurysm until his condition had stabilized. After the bilateral nephrectomy, we initiated mechanical ventilation, continuous hemodialysis, and hemoperfusion treatment with polymyxin B-immobilized fiber (PMX-DHP; Toray Medical Co., Tokyo, Japan).

On postoperative day 3 , we began gradually tapering the doses of dopamine and noradrenaline. On postoperative day 5 , we transitioned from continuous hemodialysis to intermittent hemodialysis. A culture of the samples taken from the infected region and four consecutive blood cultures revealed $C$. septicum. On postoperative day 6, we performed extubation, after which his condition gradually improved. However, on postoperative day 7 , the onset of respiratory muscle fatigue necessitated reintubation. We resumed dopamine on day 9. We planned to operate on the aortic aneurysm; however, his condition deteriorated rapidly. On postoperative day 10, $1500 \mathrm{~mL}$ of blood was lost within approximately 20 minutes from the drain; as a consequence, he died. The cause of the hemorrhage was considered to be an aortic rupture. An autopsy was not performed.

\section{Discussion}

In the present case, $C$. septicum was thought to have entered the blood from a gastrointestinal tumor, infected the aorta, and spread from the aorta to the kidneys. However, we were not certain whether this case was associated with cancer.

We searched the Embase ${ }^{\oplus}$, MEDLINE, Web of Science, and Google Scholar databases from their inception until 31 December 2015, for case reports (English language articles only) regarding C. septicum-related aneurysms. We used the following search terms: 'Clostridium septicum' and 'aneurysm' or 'aortitis.' Two reviewers (F.I. and R.I.) independently screened the 
study titles and abstracts to identify relevant articles for inclusion. The reference lists of the selected articles were also examined for additional publications suitable for inclusion. A consensus for discrepancies between the two reviewers was reached through a discussion with a third reviewer (K.S.). We excluded cases without evidence of an aneurysm with C. septicum, cases without an association with $C$. septicum, and academic conference abstracts. The earliest report found in our search was published in 1981.

The search yielded 61 articles, and 10 articles were excluded: two non-English articles (one in French and one in Spanish), five academic society presentations, and three articles that were not case reports). Of the remaining 51 articles, we excluded two involving post-endovascular aortic repair (EVAR) infection $[8$, 9], one involving post-axillobifemoral bypass infection [10], one involving mycotic endocarditis following the reconstructive treatment of congenital heart disease [11], three involving the mycotic aneurysm of arteries other than the aorta [12-14], and one involving cellulitis [15], leaving us with 43 articles. In addition to our case, we found a total of 46 cases (Fig. 2).

\section{Summary of results}

A summary of all case reports identified is presented in Table $1[6,7,16-56]$. The 6 -month mortality rate associated with $C$. septicum-related aneurysms was $79.5 \%$; of the cases, $92.6 \%$ showed the presence of periaortic gas, and surgery for a mycotic aneurysm was performed in $52.2 \%$ of the cases. The 6-month mortality rate was $66.7 \%$ for cases in which elective surgery was not possible and sufficient antimicrobial agents could therefore not be administered prior to surgery. Among the cases in which elective surgery and sufficient preoperative administration of antimicrobial agents were possible, the 6-month mortality rate was $33.3 \%$. In cases in which surgery was not performed, the 6-month mortality rate was $100 \%$.

\section{Sites of aortic aneurysm formation}

Aneurysms formed in the ascending aorta/arch in six cases $[17,18,25,27,51,56]$, only the arch in four cases [41, 42, $52,54]$, the arch/descending aorta in two cases $[19,53]$, the descending thoracic aorta in four cases $[7,23,26,46]$, and the abdominal aorta in five cases [37, 38, 48-50]. The aneurysms also involved the thoracoabdominal aorta in six cases [24, 30, 33, 43, our case, 55], the infrarenal aorta in 13 cases $[6,16,20,28,29,31,32,34-37,44,47]$, the juxtarenal aorta in four cases $[6,21,39,40]$, both the descending thoracic aorta and the juxtarenal aorta in one case [22], and an unknown location in one case [45].

\section{Operative procedures}

Of the 24 cases in which surgery was performed, in situ grafts were performed in 9 cases [19, 23-25, 30, 35, 39, 40, 49], axillobifemoral bypass in nine cases [6 (two cases), 16, $20,36,37,41,42,47]$, both in situ grafts and axillobifemoral bypass in one case [22], right axillofemoral and femoralfemoral bypass in one case [50], EVAR in two cases [54, 56], and unknown types of procedure in two cases [29,31].

\section{Prognosis according to operative procedure}

The 6-month mortality was $66.7 \%$ in cases in which axillobifemoral bypass was performed and $44.4 \%$ in cases in which in situ graft was performed.

\section{Antimicrobial agents}

After surgery, antimicrobial agents were administered for 20 of the cases $[6,16,19,20,22-24,29-31,37,39-42,47,49$,

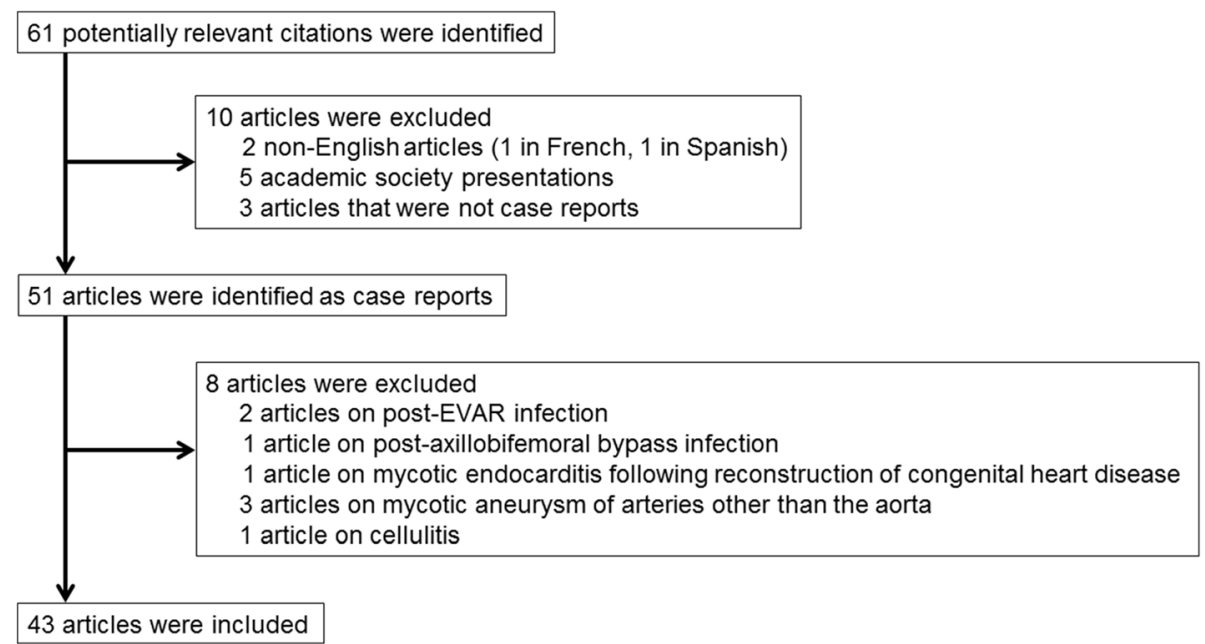

Fig. 2 Procedures used for selection of case reports. EVAR endovascular aortic repair 


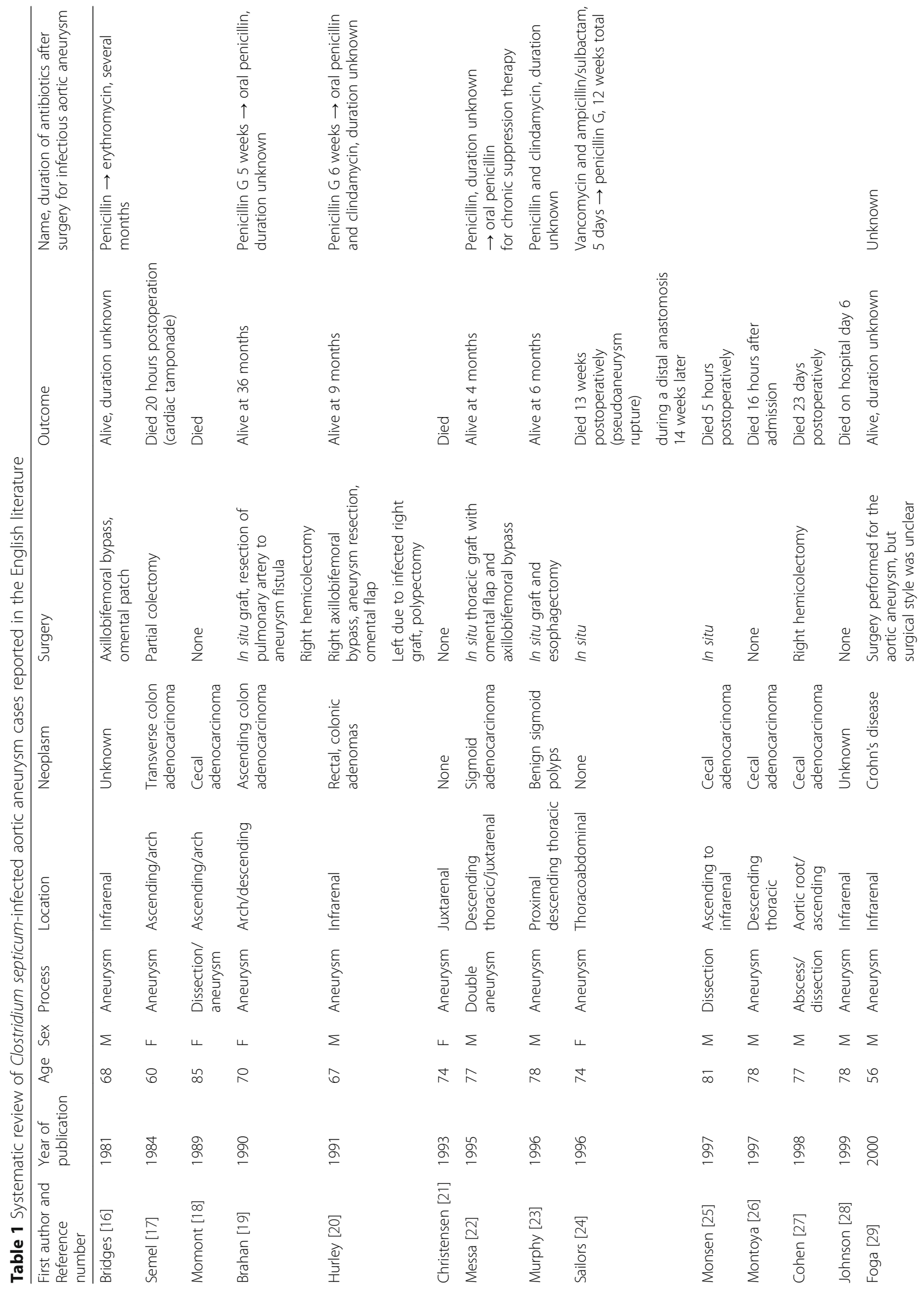




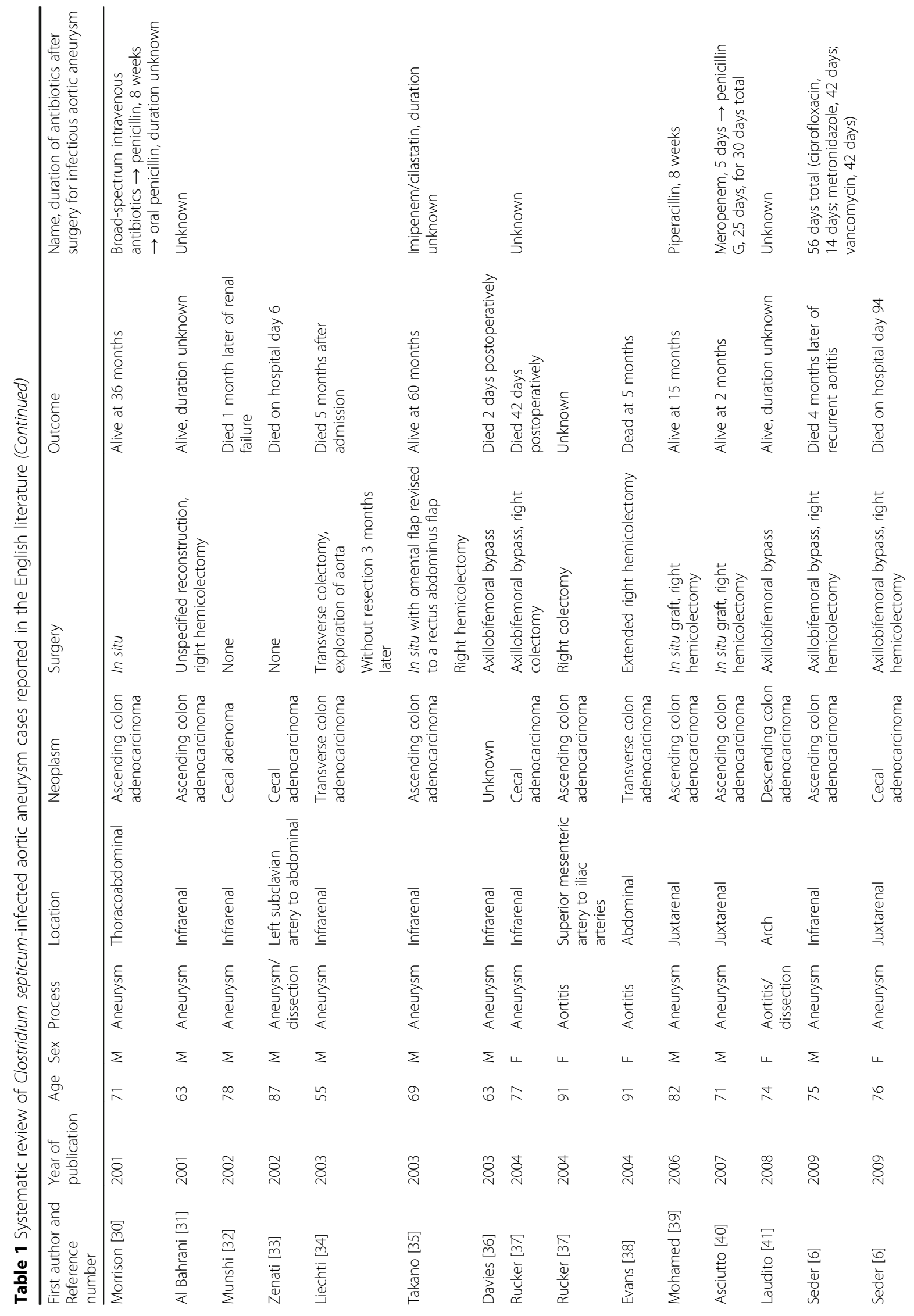




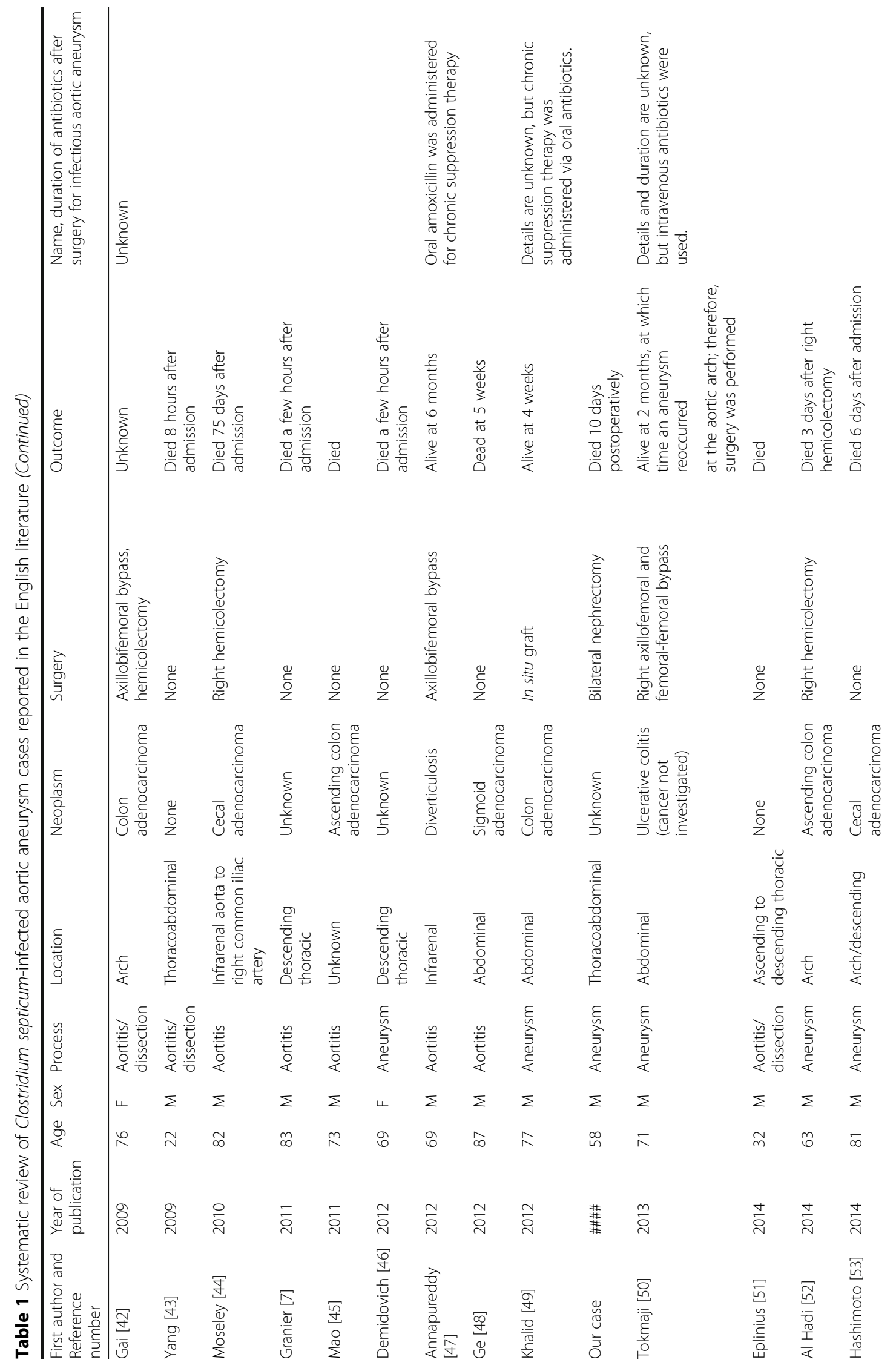




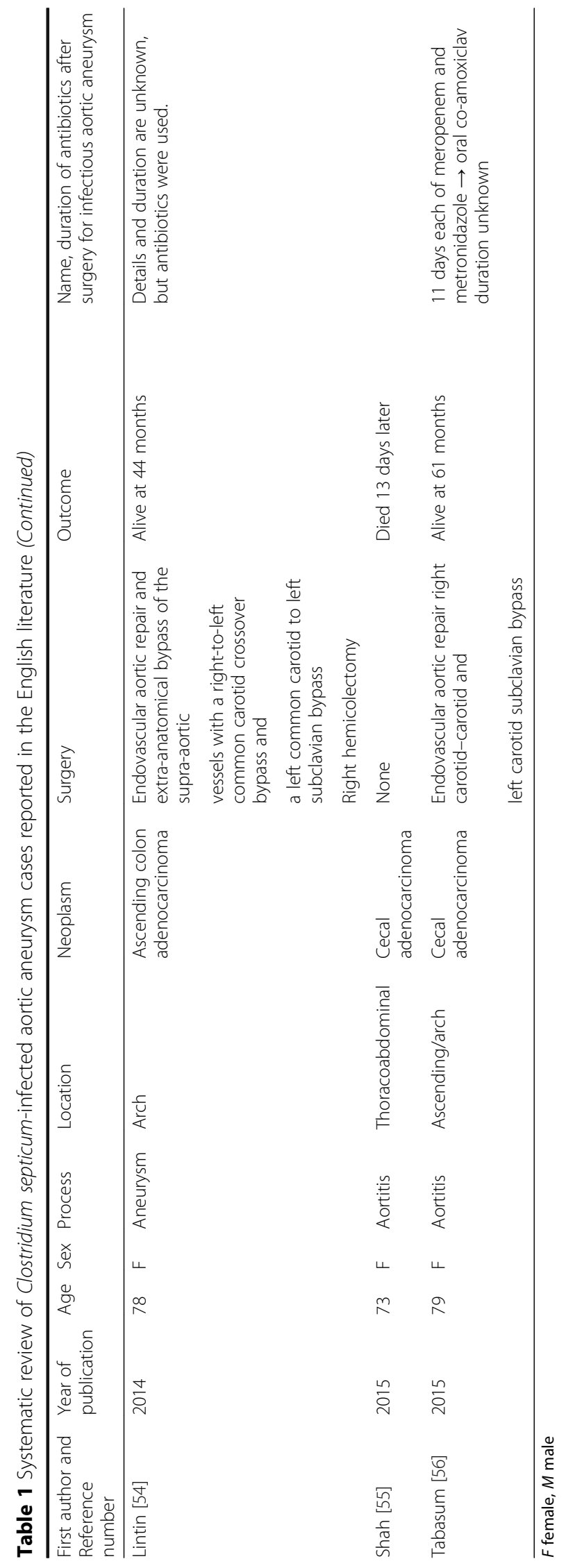


$50,54,56]$; penicillin was administered in eight of these cases. However, the duration and dosage of antimicrobial agents were unknown $[16,19,20,22,23,29,31,37,40,41$, $42,47,49,50,54,56]$.

Based on the collective evidence from our case and the published literature, we found that the associated 6month mortality was extremely high, periaortic gas was present in almost all of the cases, and no standard operative procedure had been established. It was also found that no algorithm for administering antimicrobial agents had been established, and C. septicum is associated with cancer in $82.5 \%$ of the cases. To improve mortality rates, we propose what are currently considered optimal treatments.

\section{Early diagnosis in the emergency department}

We found that the presence of periaortic gas is frequently concomitant with $C$. septicum-infected aortic aneurysms; thus, the presence of periaortic gas is probably very useful for early diagnosis. C. septicum-infected aortic aneurysm progresses quickly, with the aneurysm often expanding within days or weeks; therefore, longer delays before diagnosis result in higher mortality rates $[6,7,35,52-54,56]$.

However, infected aortic aneurysms are generally rare, accounting for only 0.5 to $1.3 \%$ of all aneurysms [57-60]. Infected aortic aneurysms do not present with characteristic findings, making them difficult to diagnose. In addition, the causative agent is chiefly Staphylococcus aureus or Salmonella; infection by $C$. septicum is even rarer. However, as shown in Fig. 1, C. septicum-infected aortic aneurysms also present with periaortic gas in a high proportion (92.6\%) of cases, allowing for their early diagnosis. Even in cases without an aortic aneurysm, periaortic gas is sometimes observed, indicating its potential to enable an even earlier diagnosis [37, 38, 44, 47, 48]. Thus, the presence of periaortic gas on a CT scan is a very important sign in this regard.

\section{Association with cancer}

In the cases we reviewed, C. septicum-infected aortic aneurysm was associated with cancer in $82.5 \%$ of cases, which is higher than the $75 \%$ of cases (35\% colorectal and $40 \%$ hematological) in which C. septicum infection had been associated with malignancy, as observed from the results of an earlier report [4]. C. septicum proliferates in tissues with a low $\mathrm{pH}$, elevated lactate levels, and low oxygen. These conditions also support the development of ulcerative lesions of the gastrointestinal tract, especially colon cancer, or colitis due to leukemia, neutropenia, and chemotherapy. Infected aortic aneurysms are believed to occur when $C$. septicum enters the bloodstream and triggers a distant infection after adhering to the aortic wall, leading to the assumption of an association with gastrointestinal cancer $[5,23]$. Therefore, it is necessary to conduct investigations for gastrointestinal cancer in addition to eradicating the source of $C$. septicum.

\section{Prognosis according to operative procedure}

We found that a standard operative procedure has not been established for the management of C. septicum-infected aortic aneurysms. If surgery is performed to treat only the infected aortic aneurysm, the gastrointestinal cancer (the source of $C$. septicum) remains, increasing the risk of reinfection and infection in other sites. Therefore, surgery should be simultaneously performed to treat both the infected aortic aneurysm and cancer, if any. In patients with an unstable condition who cannot undergo simultaneous surgery, failure to perform surgery for the infected aortic aneurysm results in a 6month mortality rate of $100 \%$; therefore, the infected aortic aneurysm should be treated first.

First, debridement of the focus of the infection (sufficient removal and cleansing of the aneurysmal wall) should be performed. The next step is revascularization; in our review, the 6-month prognosis was $55.5 \%$ for an in situ graft versus $28.6 \%$ for an axillobifemoral bypass, indicating that an in situ graft is the superior option. In general, extra-anatomic revascularization of infected aortic aneurysms, such as via an axillobifemoral bypass, has the advantage of not bringing foreign material into the focus of the infection. However, complications frequently occur, including aortic stump blowout (20\%), lower limb amputation due to bypass failure (thromboembolism; 20 to 29\%), and reinfection (20\%) [61]. In addition, mortality rates following operations for infected aortic aneurysm with anatomic revascularization and extra-anatomic revascularization are reportedly $33 \%$ and $40 \%$, respectively; thus, anatomic reconstruction yields better outcomes [59]. Many reports have also demonstrated favorable results with in situ anatomic revascularization [61-64].

With regards to graft selection, homografts are reportedly superior to prosthetic grafts in inhibiting infection $[65,66]$. Also, occlusion and deliquescence occurred in $17 \%$ of the cases during the mean 3-year follow-up period following homograft transplantation in a previous study; reoperation for both were reported to be simple, regardless of the infection [65]. The use of rifampinsoaked prosthetic grafts at the infection site has also been reported $[67,68]$.

Next, we discuss grafts with omental coverage. The omentum, a layer of tissue rich in intraperitoneal vessels and adipose tissue, processes foreign bodies at its milky spots (clusters of monocytes and lymph nodes in the vascular wall); this action is considered 
to be effective for containing infections. Omental coverage of the area surrounding an in situ prosthetic graft and homograft transplantation can prevent the recurrence of infection [63,69].

For high-risk surgical patients, EVAR is also a viable option [70, 71]. A retrospective European multicenter study found that the 6-month survival rate was 76 to $86 \%$ in the case of EVAR for an infected aortic aneurysm; this was lower for non-Salmonella infections [72]. Favorable outcomes were obtained via surgery for C. septicum-infected aortic aneurysms that involved a combination of EVAR and extra-anatomical bypass of the supra-aortic vessels with a right-to-left common carotid crossover bypass and a left common carotid to left subclavian bypass, followed by surgery for colorectal cancer [54]. A study on surgery for $C$. septicum-infected aortic aneurysm, that involved a combination of EVAR and right carotid-carotid and left carotid-subclavian bypass, followed by surgery for colorectal cancer, yielded a favorable outcome [56]. Thus, a combination of EVAR and surgery for gastrointestinal cancer might improve the prognosis. The outcome in the present case might have been favorable if EVAR was performed.

\section{Antimicrobial agents}

According to the literature, an antimicrobial agent treatment strategy has not yet been established.

\section{After surgery for an infected aortic aneurysm}

Postoperatively, antimicrobial treatment must be continued. If the aneurysmal wall culture results are known, the range of antimicrobial agents should be narrowed based on the sensitivity patterns of the culture results. The intravenous administration of antimicrobial agents needs to be continued for 6 to 8 weeks following surgery to eliminate an inflammatory response. However, the appropriate duration of subsequent antimicrobial treatment has not yet been established. Although one report stated that postoperative antimicrobial agents administered intravenously could safely be discontinued when the inflammatory response completely subsides [73], the general consensus is that the oral administration of antimicrobial agents should be continued for the rest of the patient's life (chronic suppression therapy) [58, 74]. In a previous report, despite 12 weeks of antimicrobial agent administration following surgery for a C. septicum-infected aortic aneurysm, infection recurred 5 weeks later [24]. Therefore, we support the guideline that the oral administration of antimicrobial agents needs to be continued for the rest of the patient's life.

\section{Prior to surgery for an infected aortic aneurysm}

Some authors suggest that surgery should be performed after the infection has subsided [60]. Among the 24 cases of C. septicum-infected aortic aneurysm in our review, in which surgery was performed, there were nine cases in which elective surgery and sufficient preoperative administration of antimicrobial agents were performed $[6,20,22,35,36,39,47,54,56]$. The 6-month prognosis in these cases was relatively favorable, at $66.7 \%$. It might be advisable to administer sufficient quantities of antimicrobial agents before performing surgery (at least until the negative conversion of the blood culture is confirmed). This may be done in cases where emergency surgery is unnecessary, such as cases in which infected aortic aneurysm is suspected, but aneurysm formation is either absent or small (diameter $<5.5 \mathrm{~cm}$ ) or in cases without pain or other symptoms potentially attributable to an aneurysm. The long-term administration of antimicrobial agents can greatly extend survival periods [35, 54]. Of course, as previously stated, aneurysms form rapidly and require careful monitoring and constant preparedness to perform surgery at any time.

\section{Steps to take when C. septicum is isolated in the blood culture}

If there is no contamination, a detailed examination is necessary to determine the presence of an infected aortic aneurysm as well as the presence of gastrointestinal cancer. Even when an infected aortic aneurysm is not detected, careful monitoring for the possible occurrence of an infected aortic aneurysm is necessary if there is no other identifiable cause of the bacteremia.

\section{Limitations}

There are a number of limitations to this study. First, since case reports often involve patients who survive, and we excluded academic conference abstracts, this might have been a source of bias. Therefore, the mortality rate for C. septicum-infected aortic aneurysm may have been underestimated. Second, due to the small number of cases, we were unable to assess the long-term prognosis. Long-term prognosis was reported for two patients [35, 54], both of whom underwent elective surgery and received antimicrobial agents for 25 and 41 days prior to surgery. Both cases underwent cancer excision: one case had omental graft coverage [35], and the other case underwent EVAR [54]. These patients survived for 60 months and 44 months, respectively. Given the frequency of comorbid malignant gastrointestinal tumors, longterm prognosis remains an issue for future studies. 


\section{Conclusions}

C. septicum-infected aortic aneurysm is extremely difficult to treat. However, in this review, we advocate for the need to make an early diagnosis through the identification of periaortic gas, as well as effective treatment options. We believe these could reduce mortality rates.

\section{Abbreviations}

$\mathrm{CT}$ : Computed tomography; EVAR: Endovascular aortic repair

\section{Acknowledgements}

We would like to acknowledge Tetsuya Sato, Itaru Saito, and Tetsu Sasaki for their assistance with patient management. This case was presented as a poster at the Japanese Society of Intensive Care Medicine. We would like to thank Editage (www.editage.jp) for English language editing.

\section{Funding}

No funding was received to support this work.

\section{Availability of data and materials}

Not applicable.

\section{Authors' contributions}

$\mathrm{FI}, \mathrm{KH}, \mathrm{TI}, \mathrm{AM}, \mathrm{YK}, \mathrm{HY}, \mathrm{MN}$, and $\mathrm{KS}$ contributed to patient management. FI and RI drafted the initial manuscript. TI, MN, and AM contributed to writing the report. KS critically reviewed the manuscript. All the authors have provided written consent for publication. All authors read and approved the final manuscript

\section{Ethics approval and consent to participate} Not applicable.

\section{Consent for publication}

Written informed consent was obtained from the patient's next of kin for publication of this case report and any accompanying images. A copy of the written consent is available for review by the Editor-in-Chief of this journal.

\section{Competing interests}

The authors declare that they have no competing interests.

\section{Publisher's Note}

Springer Nature remains neutral with regard to jurisdictional claims in published maps and institutional affiliations.

\section{Author details}

'Department of Emergency and Critical Care Medicine, Ohta Nishinouchi Hospital, 2-5-20 Nishinouchi, Koriyama, Fukushima 963-8558, Japan. ${ }^{2}$ Department of Emergency and Critical Care Medicine, JR General Hospital, 2-1-3 Yoyogi, Shibuya-ku, Tokyo 151-8528, Japan. ${ }^{3}$ Department of Emergency and Critical Care Medicine, The University of Tokyo Hospital, 7-3-1 Hongo, Bunkyo-ku, Tokyo 113-8655, Japan. ${ }^{4}$ Department of Infectious Diseases, Okinawa Chubu Hospital, 281 Miyazato, Uruma, Okinawa 904-2293, Japan.

Received: 11 April 2017 Accepted: 8 August 2017

Published online: 21 September 2017

\section{References}

1. Kennedy CL, Krejany EO, Young LF, et al. The a-toxin of Clostridium septicum is essential for virulence. Mol Microbiol. 2005;57:1357-66.

2. Alpern RJ, Dowell Jr VR. Clostridium septicum infections and malignancy. JAMA. 1969;209:385-8.

3. de Virgilio C, Klein S, Chang L, et al. Clostridial bacteremia: implications for the surgeon. Am Surg. 1991;57:388-93.

4. Kornbluth AA, Danzig JB, Bernstein LH. Clostridium septicum infection and associated malignancy. Report of 2 cases and review of the literature. Medicine (Baltimore). 1989;68:30-7.

5. Jendrzejewski JW, Jones SR, Newcombe RL, et al. Nontraumatic clostridial myonecrosis. Am J Med. 1978;65:542-6.
6. Seder CW, Kramer M, Long G, et al. Clostridium septicum aortitis: report of two cases and review of literature. J Vasc Surg. 2009;49:1304-9.

7. Granier M, Granier A, Fraga J, et al. Emphysematous infectious aortitis: a dramatic evolution. Eur Heart J. 2011;32:2085.

8. Halak M, Heldenberg E, Silverberg D, et al. Clostridium septicum postendovascular aneurysm repair stent-graft infection. Vascular. 2012;20:104-6.

9. Colwick SE, Alkhoury F, Martin JT, et al. Abdominal aortic aneurysm repair complicated by infection with Clostridium septicum. Ann Vasc Surg. 2011;25:839. e11-3.

10. Upchurch Jr GR, Clair DG, Whittemore AD, et al. Clostridium septicum bacteremia associated with aortic graft infection. J Vasc Surg. 1995;22:493-5.

11. Fisker N, Søgaard P. Early Clostridium septicum endocarditis and myocarditis after reconstruction of congenital heart disease. Clin Microbiol Infect. 1998;4:533-5

12. Narula A, Lake SP, Baker AR, et al. Mycotic aneurysm of the popliteal artery following right hemicolectomy. Postgrad Med J. 1988;64:638-9.

13. Asplund MW, Molinaro A. Infected solitary iliac artery aneurysm. J Vasc Surg. 1990;12:219-20.

14. Kaufman JL, Fereshetian A, Chang B, et al. Septicemia presenting with endoaneurysmal gas: CT demonstration. AJR Am J Roentgenol. 1988:151:287-8.

15. El Sayad M, Chikate A, Ramesh B. Gas gangrene presenting with back pain. BMJ Case Rep. 2014. doi:10.1136/bcr-2013-010241.

16. Bridges RA, McTamaney JP, Barnes RW. Recognition and management of ruptured infected aneurysm of the abdominal aorta. Vasc Endovasc Surg. 1981;15:360-6.

17. Semel L, Aikman WO, Parker Jr FB, et al. Nontraumatic clostridial myonecrosis and mycotic aneurysm formation. N Y State J Med. 1984;84:195-6.

18. Momont SL, Overholt EL. Aortitis due to metastatic gas gangrene. Wis Med J. 1989:88:28-30.

19. Brahan RB, Kahler RC. Clostridium septicum as a cause of pericarditis and mycotic aneurysm. J Clin Microbiol. 1990;28:2377-8.

20. Hurley L, Howe K. Mycotic aortic aneurysm infected by Clostridium septicum - a case history. Angiology. 1991:42:585-9.

21. Christensen J, Bistrup C. Case report: emphysematous pyelonephritis caused by Clostridium septicum and complicated by a mycotic aneurysm. $\mathrm{Br} \mathrm{J}$ Radiol. 1993;66:842-3.

22. Messa 3rd CA, Kulkami M, Arous E. Double clostridial mycotic aneurysms of the aorta. Cardiovasc Surg. 1995;3:687-92.

23. Murphy DP, Glazier DB, Krause TJ. Mycotic aneurysm of the thoracic aorta caused by Clostridium septicum. Ann Thorac Surg. 1996;62:1835-7.

24. Sailors DM, Eidt JF, Gagne PJ, et al. Primary Clostridium septicum aortitis: a rare cause of necrotizing suprarenal aortic infection. A case report and review of the literature. J Vasc Surg. 1996:23:714-8.

25. Monsen $T$, Palmgren $H$, Arnerlöv $C$, et al. Aortic dissection due to Clostridium septicum infection. Eur J Vasc Endovasc Surg. 1997:13:517-8.

26. Montoya FJ, Weinstein-Moreno LF, Johnson CC. Mycotic thoracic aneurysm due to Clostridium septicum and occult adenocarcinoma of the cecum. Clin Infect Dis. 1997;24:785-1007.

27. Cohen CA, Almeder LM, Israni A, et al. Clostridium septicum endocarditis complicated by aortic-ring abscess and aortitis. Clin Infect Dis. 1998:26:495-6.

28. Johnson FE. Infected aortic aneurysm. Am J Surg. 1999;178:268.

29. Foga MM, McGinn GJ, Kroeker MA, et al. Sepsis due to Clostridium septicum: case report. Can Assoc Radiol J. 2000;51:85-9.

30. Morrison Jr RC, DiMuzio PJ, Kahn M, et al. Clostridial mycotic aneurysm of the thoracoabdominal aorta - a case report. Vasc Surg. 2001;35:303-10.

31. Al Bahrani BJ, Thomas DJ, Moylan EJ. What's causing that gas? Med J Aust. 2001;174:652

32. Munshi IA, Rhee SW, Pane T, et al. Clostridium septicum mycotic aortic aneurysm. Am J Surg. 2002;184:54-5.

33. Zenati MA, Bonanomi G, Kostov D, et al. Images in cardiovascular medicine fulminant clostridium septicum aortitis. Circulation. 2002;105:1871.

34. Liechti ME, Schöb O, Kacl GM, et al. Clostridium septicum aortitis in a patient with colon carcinoma. Eur J Clin Microbiol Infect Dis. 2003:22:632-4.

35. Takano $\mathrm{H}$, Taniguchi K, Kuki S, et al. Mycotic aneurysm of the infrarenal abdominal aorta infected by Clostridial septicum: a case report of surgical management and review of the literature. J Vasc Surg. 2003;38:847-51.

36. Davies M, Byrne J, Harvey JS. Clostridial infection of the abdominal aorta. J Am Coll Surg. 2003;197:331. 
37. Rucker CM, Menias CO, Bhalla S, et al. Clostridium septicum infrarenal aortitis secondary to occult cecal adenocarcinoma. AJR Am J Roentgenol. 2004;183:1316-8.

38. Evans LT, Chey WD. Clostridial aortitis and colon cancer. Gastrointest Endosc. 2004;60:803.

39. Mohamed HK, Elliot BM, Brothers TE, et al. Suprarenal Clostridium septicum aortitis with rupture and simultaneous colon cancer. Ann Vasc Surg. 2006; 20:825-9.

40. Asciutto G, Geier B, Marpe B, et al. A case of contained ruptured aortitis due to Clostridium septicum infection in a patient with a colon malignancy. Chir Ital. 2007:59:743-6.

41. Laudito A, Gai V, Battista S, et al. Images in cardiovascular medicine. Clostridium septicum arch aortitis. Circulation. 2008;117:1609.

42. Gai V, Battista S, Bonino L. Electronic clinical challenges and images in Gl. Clostridium septicum arch aortitis. Gastroenterology. 2009;136:e4-5.

43. Yang Z, Reilly SD. Clostridium septicum aortitis causing aortic dissection in a 22-year-old man. Tex Heart Inst J. 2009:36:334-6.

44. Moseley B, Mwirigi NW, Bowen J. Clostridium septicum aortitis and cecal adenocarcinoma. Case Rep Med. 2010;2010:121728.

45. Mao E, Clements A, Feller E. Clostridium septicum sepsis and colon carcinoma: report of 4 cases. Case Rep Med. 2011;2011:248453.

46. Demidovich J, Condoluci M, Fussa M, et al. Photo quiz: a 69-year-old woman with persistent back pain. Clin Infect Dis. 2012:55(254):301-2.

47. Annapureddy N, Agarwal SK, Kanakadandi V, et al. Clostridium septicum aortitis in a patient with extensive atheromatous disease of the aorta. J Infect Chemother. 2012;18:948-50.

48. Ge SP, de Virgilio C. Clostridium septicum aortitis with associated sigmoid colon adenocarcinoma. Ann Vasc Surg. 2012;26:280. el-4.

49. Khalid M, Lazarus R, Bowler IC, et al. Clostridium septicum sepsis and its implications. BMJ Case Rep. 2012. doi:10.1136/bcr-2012-006167.

50. Tokmaji G, Gosev I, Kumamaru KK, et al. Mycotic aneurysm of the aortic arch presenting with left vocal cord palsy. Ann Thorac Surg. 2013;96:302-5.

51. Eplinius F, Hädrich C. Acute aortic dissection caused by Clostridium septicum aortitis. Forensic Sci Int. 2014;244:e38-41.

52. Al Hadi HI, Patel G, Rees MD. A rare case of Clostridium septicum mycotic aortic arch aneurysm following open right hemicolectomy for colorectal cancer. BMJ Case Rep 2014;2014. doi:10.1136/bcr-2014-204636.

53. Hashimoto T, Okamatsu $Y$, Hyakuna $Y$, et al. Perianeurysmal emphysema: Clostridium septicum-infected aortic aneurysm. Circulation. 2014;129:1900-1.

54. Lintin $L$, Wheeler $R$, Whiston $R$, et al. Mycotic thoracic aortic arch aneurysm from haematogenous spread of Clostridium septicum due to metastatic colorectal cancer: a survival guide. J Surg Case Rep 2014; 2014. doi:10.1093/jscr/rju117.

55. Shah S, Whitehead D, Sampath K, et al. A case of Clostridium septicum aortitis with concomitant adenocarcinoma of the cecum. ACG Case Rep J. 2015;2:230-2.

56. Tabasum A, Bleehen $R$, Healy $B$, et al. An atypical presentation of colonic malignancy: Clostridium septicum aortitis. BMJ Case Rep. 2015 doi:10.1136/bcr-2015-209437.

57. Klontz KC. Frequency of infected aneurysms among patients in department of veterans affairs hospitals, 1986-1990: the role of Salmonella. Mil Med. 1997;162:766-8.

58. Chan FY, Crawford ES, Coselli JS, et al. In situ prosthetic graft replacement for mycotic aneurysm of the aorta. Ann Thorac Surg. 1989;47:193-203.

59. Müller BT, Wegener OR, Grabitz K, et al. Mycotic aneurysms of the thoracic and abdominal aorta and iliac arteries: experience with anatomic and extraanatomic repair in 33 cases. J Vasc Surg. 2001;33:106-13.

60. Miller DV, Oderich GS, Aubry MC, et al. Surgical pathology of infected aneurysms of the descending thoracic and abdominal aorta: clinicopathologic correlations in 29 cases (1976 to 1999). Hum Pathol. 2004;35:1112-20.

61. Cordero Jr JA, Darling 3rd RC, Chang BB, et al. In situ prosthetic graft replacement for mycotic thoracoabdominal aneurysms. Am Surg. 1996;62:35-9.

62. Hsu RB, Tsay YG, Wang SS, et al. Surgical treatment for primary infected aneurysm of the descending thoracic aorta, abdominal aorta, and iliac arteries. J Vasc Surg. 2002;36:746-50.

63. Fichelle JM, Tabet G, Cormier P, et al. Infected infrarenal aortic aneurysms: when is in situ reconstruction safe? J Vasc Surg. 1993;17:635-45.

64. Ting AC, Cheng SW, Ho P, et al. Surgical treatment of infected aneurysms and pseudoaneurysms of the thoracic and abdominal aorta. Am J Surg. 2005;189:150-4.
65. Lesèche G, Castier Y, Petit MD, et al. Long-term results of cryopreserved arterial allograft reconstruction in infected prosthetic grafts and mycotic aneurysms of the abdominal aorta. J Vasc Surg. 2001;34:616-22.

66. Vogt PR, Brunner-La Rocca HP, Carrel T, et al. Cryopreserved arterial allografts in the treatment of major vascular infection: a comparison with conventional surgical techniques. J Thorac Cardiovasc Surg. 1998;116:965-72.

67. Gupta AK, Bandyk DF, Johnson BL. In situ repair of mycotic abdominal aortic aneurysms with rifampin-bonded gelatin-impregnated Dacron grafts: a preliminary case report. J Vasc Surg. 1996;24:472-6.

68. Bandyk DF, Novotney ML, Johnson BL, et al. Use of rifampin-soaked gelatinsealed polyester grafts for in situ treatment of primary aortic and vascular prosthetic infections. J Surg Res. 2001;95:44-9.

69. Gouny P, Valverde A, Vincent D, et al. Human immunodeficiency virus and infected aneurysm of the abdominal aorta: report of three cases. Ann Vasc Surg. 1992;6:239-43.

70. Nashef SA, Roques F, Sharples LD, et al. EuroSCORE II. Eur J Cardiothorac Surg. 2012;41:734-45.

71. Vassileva CM, Aranki S, Brennan JM, et al. Evaluation of the Society of Thoracic Surgeons online risk calculator for assessment of risk in patients presenting for aortic valve replacement after prior coronary artery bypass graft: an analysis using the STS adult cardiac surgery database. Ann Thorac Surg. 2015:100:2109-16.

72. Sörelius K, Mani K, Björck M, et al. Endovascular treatment of mycotic aortic aneurysms: a European multicenter study. Circulation. 2014;130:2136-42.

73. Hsu RB, Chen RJ, Wang SS, et al. Infected aortic aneurysms: clinical outcome and risk factor analysis. J Vasc Surg. 2004:40:30-5.

74. Hollier LH, Money SR, Creely B, et al. Direct replacement of mycotic thoracoabdominal aneurysms. J Vasc Surg. 1993;18:477-84.

\section{Submit your next manuscript to BioMed Central and we will help you at every step:}

- We accept pre-submission inquiries

- Our selector tool helps you to find the most relevant journal

- We provide round the clock customer support

- Convenient online submission

- Thorough peer review

- Inclusion in PubMed and all major indexing services

- Maximum visibility for your research

Submit your manuscript at www.biomedcentral.com/submit

) Biomed Central 